\title{
Agents That Relate: Improving the Social Believability of Non-Player Characters in Role-Playing Games
}

\author{
Nuno Afonso and Rui Prada \\ IST-Technical University of Lisbon, INESC-ID \\ Avenida Prof. Cavaco Silva - TagusPark \\ 2744-016 Porto Salvo, Portugal \\ \{nafonso, rui.prada\}@gaips.inesc-id.pt
}

\begin{abstract}
As the video games industry grows and video games become more part of our lives, we are eager for better gaming experiences. One field in which games still have much to gain is in Non-Player Character behavior in socially demanding games, like Role-Playing Games. In Role-Playing Games players have to interact constantly with very simple Non-Player Characters, with no social behavior in most of the cases, which contrasts with the rich social experience that was provided in its traditional pen-and-paper format. What we propose in this paper is that if we create a richer social behavior in Non-Player Characters the player's gaming experience can be improved. In order to attain this we propose a model that has at its core social relationships with/between Non-Player Characters. By doing an evaluation with players, we identified that $80 \%$ of them preferred such system, affirming that it created a better gaming experience.
\end{abstract}

Keywords: role-playing games, non-player characters, artificial intelligence, social, behavior, relationship, personality, theory of mind.

\section{Introduction}

As computers became more present in our lives, they transformed from simple work machines into a means of entertainment. This created a massive industry of video games, an industry that beats sales records every year, and has a yearly growth rate higher than any other entertainment industry [2].

With the appearance of this industry, many of the games were converted from their traditional form into a digital form. One of these cases was the Role-Playing Game (RPG). In its pen-and-paper version, the RPG has a very big social component, because the players create the story as they interact with each other. However, in its transition to the digital format, the RPG was transformed, in most cases, from a multiplayer into a single-player experience where instead of interacting with other people, the player interacts with autonomous Non-Player Characters (NPCs). In computer RPGs players take the role of portraying a character and goes through the game world resolving tasks that are given to them by NPCs. The player needs to interact with 
NPCs in order to improve her/his character and progress in the story, which means that even in the digital format, the RPG still has a very big social component.

The problem is that the social component of computer RPGs is yet far from the richness of its pen-and-paper format. Usually, the NPCs are not socially deep and most of the times are simply frozen in time, repeating the same action, if any, over and over again. This fact breaks the social experience of user that in turn may break his suspension of disbelief, which is one of the major factors to achieve a successful gaming experience.

In this paper we propose a simple model that can improve the social deepness of NPCs. It is centered on the idea that NPCs establish relationships with others (including the player). Moreover, NPCs have unique personalities that together with the relationships they establish and their knowledge of the relations of the others (e.g. through a theory of mind) influence their behavior. The behavior is reflected in the dialogs NPCs generate, which among other things include the quantity of information they give to the user. The game dialogues are, therefore, adapted to the social context of the game.

The paper is organized as follows. First, we describe the state of the art of computer RPGs and some academic research that focus on the use of social relationships in autonomous characters. Then we present a description of our model followed by a description of the game that was developed as a case study. Afterwards, we present the study we performed to assess the effects of the model in players' gaming experience and finish we some conclusions.

\section{State of the Art}

In order to see what we can do to improve social behavior of NPCs in RPGs, we first took a look at how NPCs are portrayed in today's RPGs. Artificial Intelligence (AI) of the NPCs in games is usually implemented with the use of state machines, since it allows for very controlled behavior. However, by using such pre-defined behaviors, in order to attain a rich social behavior from the NPCs, there has to be an extensive authoring. In order to explore a more sophisticated way for the creation of social behavior, we looked at the academic research.

The next two sections show some of our findings on both games and academic community. Lastly, we will present a survey to RPG players in order to get their opinion on the current state of RPGs.

\subsection{Role-Playing Games}

In order to see how we can improve RPGs we analyzed a few in order to see their flaws: Neverwinter Nights, Knights of the Old Republic II, World of Warcraft, Dungeon Siege II, Fable, and The Elder Scrolls IV: Oblivion. In all of them the NPC behavior is almost the same; the player will engage with NPCs that will provide him with quests that he may or not decide to resolve. The NPCs that supply these quests to the player are usually frozen in time waiting for the player to engage them. If a player accepts the quest, s/he continues with her/his journey, and after resolving the quest returns to the NPC, which will probably still be stuck in time, to receive some kind of reward. NPCs do not have a rich social behavior. 
However, game developers are starting to take a closer look at NPCs' AI. Two examples of recent games that try to create richer NPCs are Fable [8] and The Elder Scrolls IV: Oblivion [3]. These RPGs have some features that make them standout from the rest, like some individual believability (e.g. NPCs have jobs and houses) and some social interaction (e.g. NPCs simulate conversation between each other, in Oblivion the player can even hear these conversations, although they clearly sound like tips for her/him).

Nevertheless, even these RPGs have room for improvement. Although there are social interactions, these are not meaningful. For example, in Oblivion the player can enter the house of a NPC and wake her/him up to engage in conversation, and the NPC will simply talk back, ignoring the fact that a stranger just broke-in in the middle of the night. Another situation that we encounter is that we can steal from a NPC and s/he will start calling the police, but if we engage in conversation with her/him, s/he will talk back like nothing happened.

\subsection{Academic Community}

AI in games mostly feel like finite state machines, if something happens the NPC jumps into a given state. However, in order to have more interesting emergent behavior, this approach is not effective. We decided to explore the academic research and see what ideas regarding social behavior were being explored on autonomous agents, and how they could adjust to the NPCs.

Avatar Arena [15] is a system in which a task is delegated to an autonomous character (i.e. agent). The task in this case is a negotiation between the avatars (i.e. agents that are virtual representations of the users). The idea was to create a system that resembled a human-human negotiation in which not only rational arguments but also the social context and the personalities of the negotiating parties need to be taken into account.

The authors focused on how social relationships could impact an avatar's behavior. To model social relationships between the avatars, they based in socio-physiological theories of cognitive consistency, specifically in the Heider's Balance Theory [6] and Festinger's Theory of Cognitive Dissonance [5].

To model the avatars' personality they based on the Five-Factor Model (FFM) [9] implementing three of its dimensions: Extraversion and Neuroticism because they can be found on most of the theories, and Agreeableness because it is important when dealing with social relationships.

PsychSim is a multiagent-based simulation tool for modeling interactions and influence among groups or individuals [14]. It is based on the fact that the message exchanged between two agents, like in human communication, depends not only on its content but also in the way we see the communicator. Therefore, our actions take into consideration our view of the communicator and also the reaction we expect from her/him.

This means that PsychSim revolves around the notion of Theory of Mind (ToM). Each agent has her/his own decision-theoretic model of the world including her/his beliefs about the environment and her/his beliefs about the other agents' beliefs. 
Social Role Awareness was the work of Prendinger and Ishizuka [13], in which they propose that animated agents should have a notion of social roles when interacting with other agents, and adjust their behavior accordingly.

The goal of the authors' work was to create autonomous agents that would be much more interesting conversational partners for language conversation training.

These agents have a standard theory of reasoning about emotions, the OCC model [11] and a simple model of personality that consists of two dimensions from the FFM model, Extraversion and Agreeableness, which according to André et al. [1] are essential for social interaction.

The core of the authors' work is basically a social filter. Before choosing a particular action (e.g. express an emotional state), it will go by the social filter and determine the agent's behavior based on her/his personality and social role.

The $\mu$-SIC System [10] is a social model that consists of an Artificial Neural Network (ANN), which will be responsible for determining which social interactions the character should engage with another.

The $\mu$-SIC System uses three psychological models: the Eysenck personality model [4] the Lang mood model [7], and the relationship model which has its psychological basis on the work of Wish et al. [16].

One thing to note is that this work is the first one that we encounter that focuses on games. Usually academic research is more focused on few agents interacting and try to make the interaction as close to the human interaction as they can, but this one focused on many characters like we find in games, making concessions in order to maintain a responsive real-time experience.

Prada [12] has conducted some research focusing on the problem of engaging users in a group of agents that share a common collaborative task. The believability of the interactions was a key issue, thus, he developed the Synthetic Group Dynamics Model (SDG Model) to increase the believability of the group interactions. The construction of this model was supported on several theories and results of studies of human group dynamics found on social sciences. It defines the knowledge that each individual should build about the others and about the group it belongs, and how this knowledge drives their interactions in the group. One important feature of the SGD Model is that it defines the social relations of the group's members in two different dimensions: (1) the structure of power and (2) the structure of interpersonal attraction. The dynamics of the group is supported on a set of categories that identify the possible interactions that can occur in the group. These interactions are categorized in two main classes, those that are related to the task and those that are related to the socio-emotional relations.

\subsection{RPG Gamer Survey}

To search for evidence that players, indeed, expect for better social behavior from NPCs, a pilot study was performed. This study consisted in a questionnaire that was published in the Internet and disseminated in several gamers' forums ${ }^{1}$. The questionnaire was removed one month and a half later, with 250 complete answers collected.

\footnotetext{
${ }^{1}$ Forums from: BioWare Corp, World of Warcraft Europe, GameDev-PT, GameSpot, GamSpy, Lionhead Studios, Mega Score, Obsidian Entertainment, Planet Dungeon Siege, The Elder Scrolls.
} 
The gamers' demographic was $37 \%$ between 12 and 18 years old, $39 \%$ between 19 and $25,9 \%$ between 26 and 30,10\% between 31 and 40, and 3\% over 40 years old. Of all the gamers, $93 \%$ were males, and $41 \%$ played pen-and-paper RPGs.

One of the aims of the study was to find if there was any significant difference between what players expect from the NPCs and what they found on the RPGs they play. Thus, players were asked to rank (from 1 to 4), regarding what they desire (i.e. how important they feel they are for the gaming experience) and what they found int the games they have played, some features that we believe to be relevant for NPCs' believability: personality, emotions, pro-activeness, individual memory, ability to form social relationships with the player, ability to form social relationships with other NPCs.

When comparing the answers, there was a significant difference between what players desire and what players found regarding all features: personality $(\mathrm{T}=1556.50$, $\mathrm{p}<0.001, \mathrm{r}=-0.44)$, emotions $(\mathrm{T}=1629, \mathrm{p}<0.001, \mathrm{r}=-0.36)$, pro-activeness $(\mathrm{T}=1519.50$, $\mathrm{p}<0.001, \mathrm{r}=-0.40)$, individual memory $(\mathrm{T}=1639, \mathrm{p}<0.001, \mathrm{r}=0.39)$, relationships with the player $(\mathrm{T}=1346, \mathrm{p}<0.001, \mathrm{r}=-0.43)$ and relationships with other NPCs $(\mathrm{T}=1548$, $\mathrm{p}<0.001, \mathrm{r}=-0.43$ ).

From the results above, we can see that there is still a lot to improve regarding NPCs believability. In addition, the difference seems to be higher $(\mathrm{r}>0.4)$ relatively to personality, and the ability to establish relationships with both the player and other NPCs. This supports our proposal of implementing social relationships in order to enhance the social behavior of the NPCs.

Looking at the correlations between some of the questionnaire's items, we found some interesting results. Players that played pen-and-paper RPGs seem to identify more flaws in computer RPGs regarding characters' personality $(\mathrm{N}=250, \mathrm{r}=0.14$, $\mathrm{p}<0.05)$ and pro-activeness $(\mathrm{N}=250, \mathrm{r}=0.14, \mathrm{p}<0.05)$. Also, players that play more often expected more from characters' pro-activeness $(\mathrm{N}=250, \mathrm{r}=0.16, \mathrm{p}<0.01)$ and believe more on the importance of improving social behavior of NPCs to improve the role-play experience $(\mathrm{N}=250, \mathrm{r}=0.13, \mathrm{p}<0.05)$. Finally, when asked if they believed that a richer social behavior would improve their gaming experience, $94 \%$ answered positively.

\section{Conceptual Model}

As we could see in the previous section, there is still much to improve in order to attain believable social behavior of NPCs in computer games. Not just our analysis of games tells us this, but also gamers say that they would prefer NPCs with richer social behavior. As mentioned before, we propose to improve the social behavior of NPCs by introducing a mechanism of social relationships between them. This mechanism is based on a concept model that defines for each NPC: Personality, Relationships with others, and Theory of Mind (ToM).

In Fig. 1 we can see the representation of a character's mind, which contains the concepts of personality, relationships and ToM, and how they are connected. 


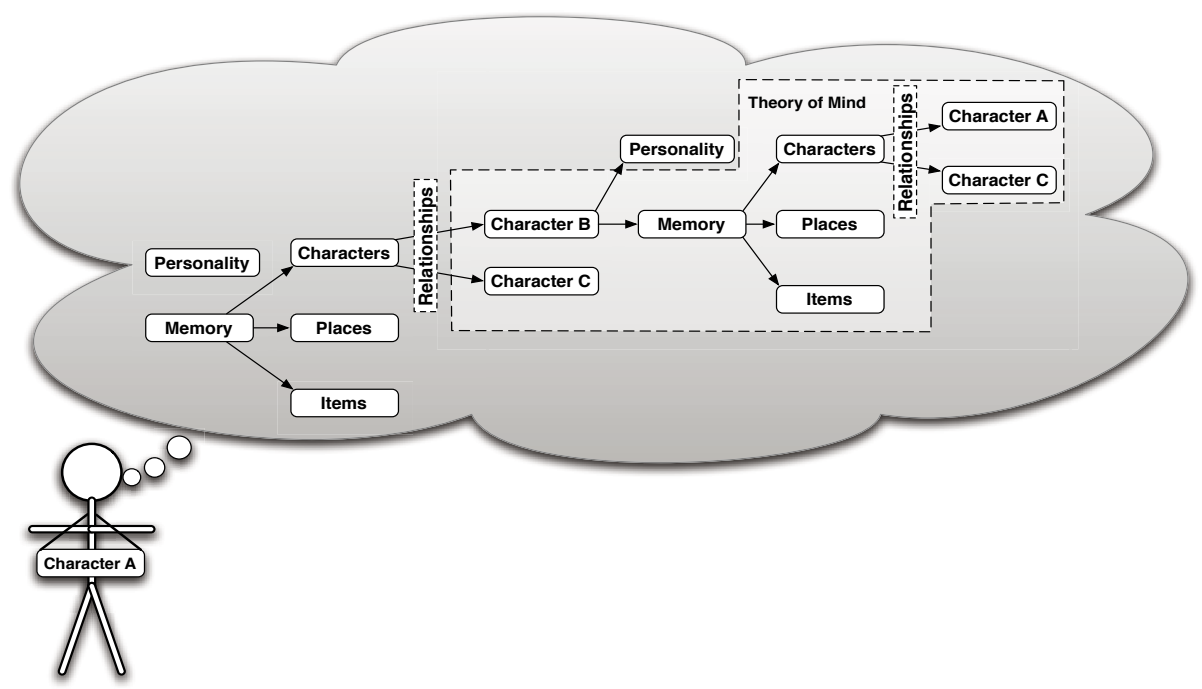

Fig. 1. Mind of Character A

In the following sections we will explain all these concepts and why we believe that all of them are necessary to improve social behavior.

\subsection{Relationships}

We consider relationships the most important concept because it represents our social connection with each person, it influences how we interact with each other, and without them there is no real social behavior. In RPGs we only have a glimpse of relationships in scripted events, like cut-scenes, which, besides being pre-defined, are scattered along the story and do not create a continuous social behavior.

Some of the recent RPGs try to give a notion of relationships. One common case is NPCs living in the same house, although we do not see behavior such as the man, which we assume that is the husband, kiss the woman before going out.

Our relationship system is based on Heider's Theory of Balance [6], in which relationships are a ternary association between two persons and a concept (see Fig. 2).

For the representation of relationship we consider a simplification of the relationship model proposed by Wish et al. [16], which may be extended in a future

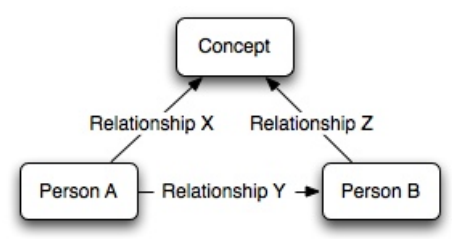

Fig. 2. Heider's Balance Theory 
version. We only consider one dimension, Likes/Dislikes axis, since we considered it to be one of the most expressive and easily understandable dimensions.

A high value in this axis can be easily understood as a positive relationship between two NPCs, and the opposite for a low value. We acknowledge that only this axis is not sufficient to accurately map the gamut of relationships, but our focus at this moment is not to accurately map relationships but instead to see if a minimum of relationships will affect the gaming experience of RPG players.

\subsection{Personality}

In real life, not everyone acts or reacts the same way. For example, if a bully starts beating up a kid in school, it is not sure that everyone will back off or stay and see the fight. Some might also engage in the fight, others might go and find help. Furthermore, not everyone will see the event the same way, some will stay on the bully's side, some will stay on the victim's side, and others will not even care about the incident. Of course there are other factors that influence where we stand, but our personality is at the core of how we might react in a given situation.

Also in games, personality is one of the main differences between the primary (e.g. plot/quest related) and secondary NPCs. It is not common to find a primary NPC's personality in another NPC, but the personalities of secondary characters appear quite often replicated (e.g. all the merchants have the same personality).

We based our concept of personality on a simplified version of the FFM [9], only considering one of its dimensions, Agreeableness. We chose only this dimension for the same reasons that we chose only one dimension to represent relationships, we want a dimension that is easy to understand and measure.

Again, we do not consider that only this dimension is suitable to represent personality, and we believe that it would have to at least include the dimensions present in Eysenck model [4], Extraversion and Neuroticism, since there seems to be a consensus that they are fundamental in implementing relationships [1] [10] [15].

\subsection{Theory of Mind}

A ToM means that characters have a mental representation about others' beliefs.

In our conceptual model, this means that NPCs have beliefs concerning the other characters' beliefs, specifically their relationships with others. For example, John may believe that Peter likes Mary.

ToM is necessary since it is important for NPCs to be aware of each other, and only then can they have interesting emergent social behavior.

\subsection{Behavior Generation}

Our model determines two things: (1) with whom the NPC will interact and (2) the quantity of information transmitted in conversations.

The NPC decides with whom s/he will interact based on the relationship with that character, giving preference to characters that s/he likes, and try to avoid characters s/he dislikes. In each conversation, the relationships will influence the quantity of information transmitted (i.e. if they like the character they are talking to and/or the object of the conversation they will give out more information, and the opposite if 


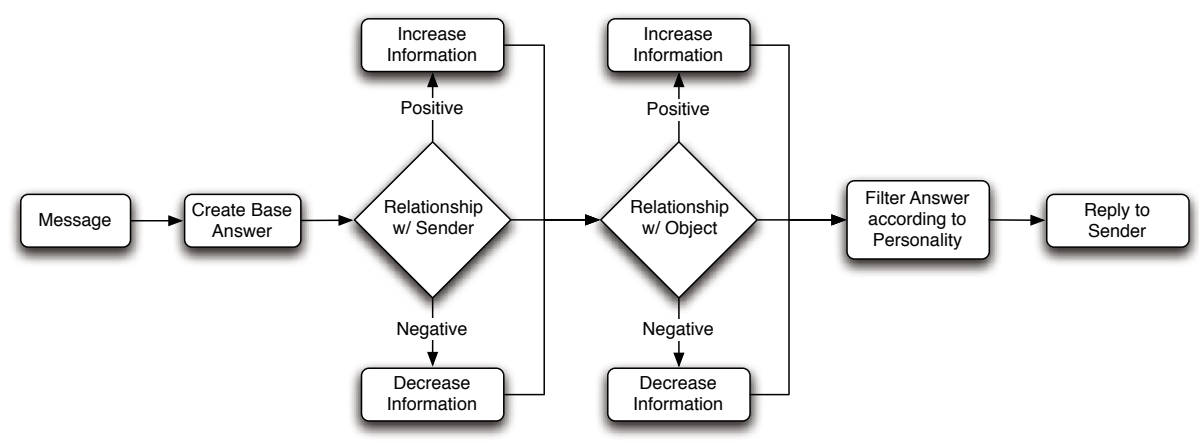

Fig. 3. How the model influences conversations

they dislike), and the personality will influence how the answers are transmitted (i.e. the text, a friendly NPC will be more polite, see Fig. 3).

\section{Case Study: Zion's Market}

In order to test our proposal we developed a small game that takes place in a market, which is a very common scenario of RPGs.

The player had a malfunction in her/his shuttle crashing on planet Zion, and s/he has to get some items to repair it. In order to complete this task, the player has to find a mechanic to tell her/him what items s/he needs, buy the items from the merchants, and then hire the mechanic to repair her/his shuttle. All these tasks are done through conversations with each of the NPCs (Fig. 4 shows the player having a conversation with an unfriendly merchant).

The game has six NPCs, three are merchants, one is a mechanic, and the other two are customers just like the user. The merchants and the mechanic are standing in their own stands waiting for customers. The two customer NPCs have the ability to enter/leave the scenario (through their shuttles) and trade items with the merchants.

\subsection{Sample Interaction}

In Fig. 4 we see an example of a conversation between the player and a merchant called John. When the player engaged John, s/he asked him "Do you know who has Energy Cells". In the top part of the figure we can see John's answer.

The first thing we notice is John's personality. By the way he talks we can see that he is rude, hence is personality has a low agreeableness value. The second thing shown in the dialog is that John mentions that he thinks that there is another merchant that sells Energy Cells, a merchant called Jeff. Finally, we can see that John does not like Jeff, since he hints the player not to go see him because "He is really not that nice".

Next the player went to Jade, a merchant that has a stand next to John. She has a neutral personality, not being rude as John, nor being very friendly. When the player asks the same question that s/he asked John, Jade will have a whole different answer. She answers that both John and Jeff sell energy cells, however, because she likes Jeff, she will try to persuade the player to go buy from him. 


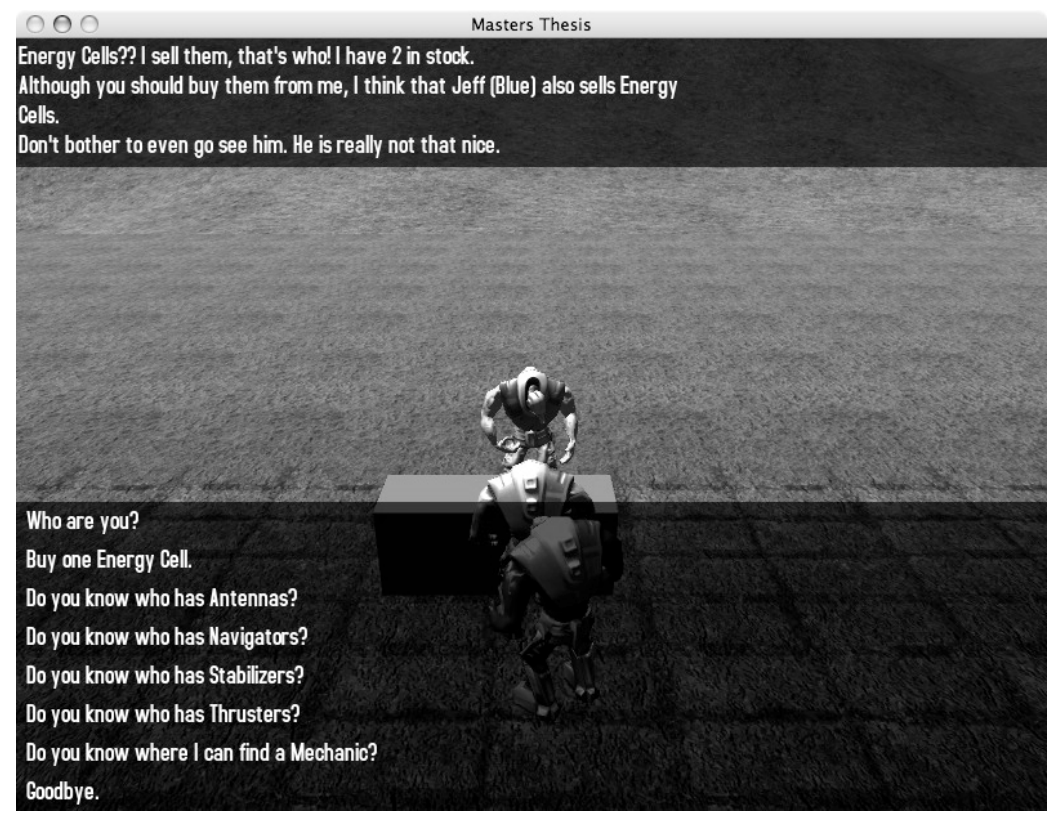

Fig. 4. Screenshot of Zion's Market

\section{User Evaluation}

This section describes the experiment that we conducted in order to test if social relationships could create a richer social behavior in NPCs, which would lead to an improved gaming experience. This experiment was conducted with the game Zion's Market presented in the last section.

The evaluation was done based on a questionnaire that was given to the users with some questions about the existence and identification of the personalities and the relationships of the NPCs. The logs created from the interaction that the player had with the NPCs (e.g. number of conversations with each one, number of items bought from each merchant) were also recorded.

Each player played the game twice: one time with the model here proposed, and the other without. The players were not told the differences between both versions.

\subsection{Method}

The evaluation was done with a total of twenty players. There was no time limit, the game ended when the player finished the objective of the game (i.e. hired a mechanic after buying all the items needed to repair the shuttle). The process was divided into 4 parts:

1. Players were given a version of the scenario to play;

2. Players were given a questionnaire about the version they played;

3. Players were then given the other version of the scenario to play;

4. Finally, players answered the same questionnaire about the second version they played. 
The whole process took between 20 to 40 minutes with each player.

The version with which the players would start with was evenly distributed, with ten of them starting with version 1 , which does not contain social relationships, and the other ten starting with version 2 , which contains social relationships, having already played the game and answered the questionnaire when they played for the second time.

The limitations of version 1 were that the characters were not aware of each other, they did not know each other (i.e. did not have memory/ToM of other NPCs), and hence they did not have relationships with each other.

Before the players started the experiment, the game's objective was explained to them. The players were not told which of the versions had social relationships.

The questionnaire had a few questions about personal information (e.g. gender, age), NPCs' personalities (if they thought that the NPCs had different personalities and what kind of personality each one had) and relationships (if they thought that the NPCs had relationships between them, and what kind of relationship was), and a question for them to rate the gaming experience of each scenario. Finally, they were asked what version they preferred.

\subsection{Results}

This section presents the analysis of the data retrieved from the questionnaire and from the logs of each version of the game that the players played.

We identified that there was a big difference between the ratings that were given to each version $(Z=3.207, p=0.001)$, with version 2 (i.e. with relationships) receiving a mean rating of 3.45 and version 1 (i.e. without relationships) receiving 2.85 .

The questionnaire showed that $80 \%$ of all players preferred version 2 . Of the players that play RPGs ( $55 \%$ of the all players) $82 \%$ of them also preferred version 2 , and hence given a difference so little between players that play different types of games and players that play RPGs, one could assume that the introduction of social relationships could improve the gaming of other types of games. One interesting fact is that $100 \%$ of the players that played version 1 first, preferred version $2(\mathrm{~N}=20, \mathrm{r}=0.5$, $\mathrm{p}<0.05)$.

We analyzed if there was a significant difference between both versions. The results show that there was a significant difference regarding the existence of relationships between the versions $(\mathrm{Z}=3.464, \mathrm{p}=0.001)$, which we were expecting since it is the differentiating factor between the two versions. In version 1, 40\% of the players answered that the NPCs had social relationships, although they would generally not remember which NPCs had them, nor what kind of relationships they were; approximately 93\%, when asked what kind of relationship was, answered that they did not know. In version 2, $100 \%$ of the users answered that the NPCs had social relationships between them.

There was a big difference between the number of relationships they identified in the merchants and the mechanic, which makes sense given the fact that the users interacted more with the merchants (a mean of 1.59 conversations with each) and the mechanic (a mean of 2.25 conversations) than with the customers (a mean of 0.82 conversations with each). 
It is also worth mentioning that the number of conversations between the player and the merchants is a bit higher in version 2, with 1.65 conversations with each merchant versus 1.53 in version 1 . The number of messages exchanged between them in conversations is really much higher in version 2 (9.67 with each NPC, versus 6.33 in version 1), as it was also expected since the players can ask information about the other NPCs.

We also found some interesting results, like the users remembering better the NPCs that had the personalities in the extremes of the personality axis (friendly $65 \%$ and unfriendly $77.5 \%$ ).

\section{Conclusions}

Clearly with the maturing of the computer games' market, game studios start doing more elaborate games. Like with the progression of graphical quality, it is only natural that developers start to focus on areas yet to release their full potential, like in the case of NPC AI.

The purpose of this work was to test the hypothesis that if we enrich the social behavior of the NPC by implementing social relationships, the player's gaming experience can be improved.

To test this hypothesis, a conceptual model was developed taking into account the concepts of relationship, personality, and theory of mind. An implementation of this model was used to develop a game that was evaluated by players.

The players played both version of the game, one with social relationships, and the other without, and in the end $80 \%$ of them preferred the version with social relationships.

Although we believe that this could be the basis for mode believable social behavior, we also believe that much is still to be done. The next step would be to expand the concept model to more accurate definitions of relationship and personality, followed by the inclusion of social roles, which we believe that are also very important when representing rich social behavior.

\section{Acknowledgments}

The work presented in this paper was supported by Mind RACES (Contract 511931) and eCircus (Contract no. IST-4-027656-STP) projects carried out with the provision of the European Community in the Framework VI Programme.

\section{References}

1. André, E., Klesen, M., Gebhard, P., Allen, S., Rist, T.: Integrating models of personality and emotions into lifelike characters. In: International Workshop of Affect in Interactions. Towards a New Generation of Interfaces (1999)

2. BBC News: US Video Games Sales Hit Record, http://news.bbc.co.uk/1/hi/business/7195511.stm 
3. Bethesda Softworks: The Elder Scrolls IV: Oblivion, http: / / www.elderscrolls.com

4. Eysenck, H.J., Rachman, S.: The Causes and Cures of Neuroses. Routledge/Kegan Paul, London (1965)

5. Festinger, L.: A Theory of Cognitive Dissonance (1957)

6. Heider, F.: The Psychology of Interpersonal Relations (1958)

7. Lang, P.J.: The Emotion Probe: Studies of motivation and attention. A study in the Neuroscience of Love and Hate (1995)

8. Lionhead Studios: Fable, http: / / fable. lionhead.com

9. McCrae, R.R., John, O.: An introduction to the five-factor model and its applications. Journal of Personality 60, 175-215 (1992)

10. Namee, B.M., Cunningham, P.: The Driven Simulation of Socially Interactive Agents (2002)

11. Ortony, A., Clore, G.L., Collins, A.: A Cognitive Structure of Emotions. Cambridge University Press, Cambridge (1988)

12. Prada, R., Paiva, A.: Believable Groups of Synthetic Characters. In: 4th International Joint Conference on Autonomous Agents and Multi Agent Systems. ACM Press, Utrecht (2005)

13. Prendinger, H., Ishizuka, M.: Social Role Awareness in Animated Agents. In: 5th International Conference on Autonomous Agents, pp. 270-277. Montréal (2001)

14. Pynadath, D.V., Marsella, S.C.: PsychSim: Modeling Theory of Mind with DecisionTheoretic Agents. In: International Joint Conference on Artificial Intelligence, pp. 1181$1186(2005)$

15. Rist, T., Schmitt, M.: Avatar Arena: an attempt to apply socio-physicological concepts of cognitive consistency in avatar-avatar negociation scenarios. In: AISB 2002 Symposium on Animated Expressive Characters for Social Interactions, London, pp. 79-84 (2002)

16. Wish, M., Deutsch, M., Kaplan, S.: Perceived Dimensions of Interpersonal Relations. Journal of Personality and Social Psychology 33(6) (1976) 